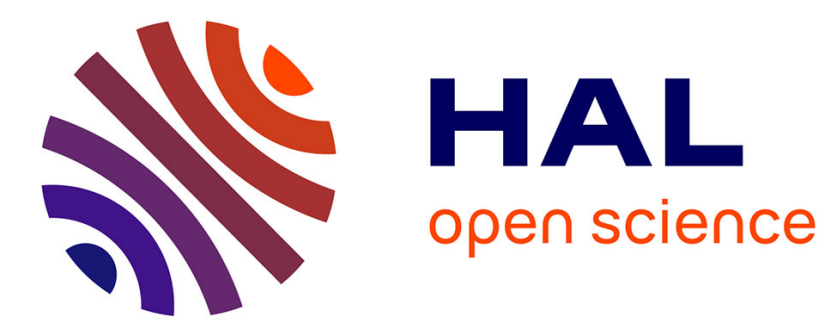

\title{
Temporal Dissection of Stimulus-Driven and Task-Driven Processes during Perceptual Decision about 3D SFM Stimuli
}

\author{
A Miskiewicz, S Buffat, J Lorenceau, A.-L Paradis
}

\section{- To cite this version:}

A Miskiewicz, S Buffat, J Lorenceau, A.-L Paradis. Temporal Dissection of Stimulus-Driven and Task-Driven Processes during Perceptual Decision about 3D SFM Stimuli. Supek, Susac. Biomag 2010, France. 28, Springer Verlag, pp.326 - 329, 2010, IFMBE Proceedings Series, 978-3-642-12196-8. 10.1007/978-3-642-12197-5_76. hal-01076912

\section{HAL Id: hal-01076912 https://hal.science/hal-01076912}

Submitted on 23 Oct 2014

HAL is a multi-disciplinary open access archive for the deposit and dissemination of scientific research documents, whether they are published or not. The documents may come from teaching and research institutions in France or abroad, or from public or private research centers.
L'archive ouverte pluridisciplinaire HAL, est destinée au dépôt et à la diffusion de documents scientifiques de niveau recherche, publiés ou non, émanant des établissements d'enseignement et de recherche français ou étrangers, des laboratoires publics ou privés. 


\title{
Temporal dissection of stimulus-driven and task-driven processes during perceptual decision about 3D SFM stimuli
}

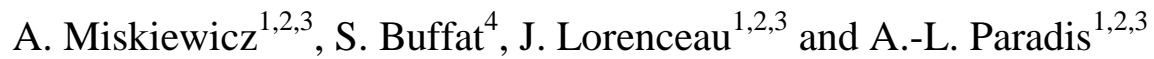 \\ ${ }^{1}$ UPMC, Centre de Recherche de L'Institut du Cerveau et de la Moëlle épinière (Cricm), Paris, France \\ ${ }^{2}$ CNRS, Cricm UMR 7225, Paris, France \\ ${ }^{3}$ Inserm, Cricm UMR-S975, Paris, France \\ ${ }^{4}$ IRBA, Brétigny-sur-Orge, France
}

\begin{abstract}
Analyzing the visual properties of a stimulus, such as its shape or its motion, involves a temporally constrained cascade of processes including sensory integration, attentional selection and perceptual decision. In natural conditions, these processing stages may temporally overlap. In this study, we used a time constrained paradigm designed to segregate these stages, and recorded MEG activity to characterize their cortical correlates.

The experimental sequence comprised a structure-frommotion (SFM) target flanked by pre and post masks, which limited the stimulus-driven processes in time.

The MEG responses were recorded in three perceptual tasks bearing on the presence or absence of the target SFM stimulus, on its direction of motion or on its 3D shape.

Subjects' responses were delayed to the end of the trials using a random stimulus-response mapping.

The analysis of the evoked magnetic fields reveals temporally non-overlapping responses for stimulus-driven and taskrelated processes.

Source reconstruction reveals that the occipital, dorsal and ventral stimulus-related areas follow different temporal profiles, The response in IPS slowly rising after the beginning of the activity in the lateral occipital cortex and falling back to the baseline prior the delayed motor response, may reflect accumulating evidence on the presented stimulus leading to a motor response, in line with the results from single cell studies in monkey LIP [1].
\end{abstract}

Keywords - visual motion, 3-D shape, perceptual decision, MEG, source reconstruction.

\section{INTRODUCTION}

Analyzing the visual properties of a stimulus, such as its shape or its motion in the 3-D environment, involves a temporally constrained cascade of processes including sensory integration, attentional selection and perceptual decision. In natural conditions, visually dynamic events require the integration of information about form and motion. Recent studies have challenged the traditional dichotomy between form- and motion-related processes in the ventral and dorsal visual pathways, and there is now evidence that form and motion processing interact in several ways. Both figural and low-level form cues guides motion perception, whereas motion cues can induce figural percept (see [2] for a review). However, more work is needed to better understand the perceptual decision stages and the attentional modulation required by the task. The experiments described in this paper aims at investigating these issues.

To segregate the different processing stages, we used structure from motion stimuli which yield both motion and shape percepts from motion cues only, and designed a time constrained paradigm with dynamical masks. We recorded MEG activity to characterize the cortical correlates of such perception in three perceptual tasks bearing on the presence or absence of a target SFM stimulus, its direction of motion or its 3D shape. We reasoned that the masks would isolate a time window for measuring the integration of form and motion cues by interrupting the early visual processing. By carefully choosing supra-threshold stimuli, we ensured that this integration yield to a perceptual decision, the MEG correlate of which should be detectable.

We specifically tested when the attentional modulation induced by the task takes place in the cortical activity, and if this modulation could be found in the occipital and intraparietal areas that are involved in the stimulus integration. We also searched a magnetic correlate of the perceptual decision processes in IPS, a region known to be involved in the accumulation of evidence in visual task implying motion stimuli [1].

\section{MAterials AND METHODS}

\section{A. Experimental procedure}

Participants. Sixteen people (age 28.5 \pm 8.1 , right-handed, normal or corrected-to-normal vision, $7 \mathrm{men}$ ) took part in the study after giving their informed consent. The study was approved by the Local Ethical Committee.

SFM stimuli and motion masks. Structure-from-motion (SFM) stimuli were made of 140 white dots $\left(0.19^{\circ}\right.$ visual angle) randomly distributed on a black background and back-projected on a 3D virtual surface. The surface had three possible shapes and oscillated in depth with three 
possible motion directions. Stimuli were viewed through a $20^{\circ}$ circular mask hiding the boundaries of the 3D surface. Overall, nothing but motion cues could be used to identify the shape.

For the masks, dots followed a random walk with instant speeds comprised between $0 \% \mathrm{~s}$ and $7 \%$ s to match that of the SFM stimuli. More details about the display can be found in [3], where identical stimuli were used.

Experimental design. During the MEG recording, participants underwent six runs of 90 trials (see Figure 1) each.

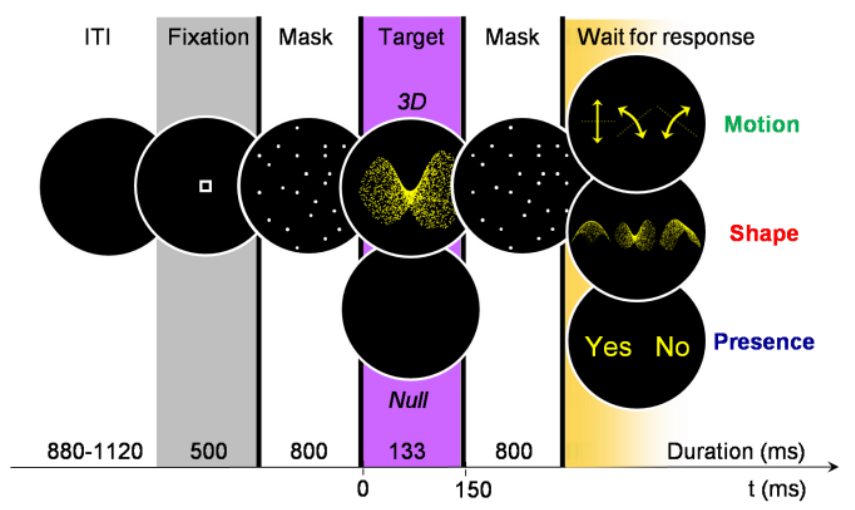

Fig. 1 Schematic time flow of a trial

Depending on the experimental run, participants had to:

- identify the target shape among three (Shape task);

- identify its motion direction among three (Motion task);

- answer whether a 3D stimulus appeared or not among the mask (Presence task). Run order was randomized across subjects.

The target, either a 3D stimulus $(56 \%)$ or a black screen (44\%) was flanked by dynamical masks to limit the duration of its visual integration. Because the stimulus-response mapping varied across trials, participants had to wait for the response screen to give their answer, so that motor activity was delayed after the end of the second mask.

Participants fixated the center of the screen but were advised to broaden their attention on the whole viewing window to ease identification. When a null target occurred in the shape and motion tasks, they had to choose a default response, which was specified at the beginning of the experiment and was randomized across subjects.

Stimulation was projected at $90 \mathrm{~cm}$ from the participants, using a calibrated LCD video device $(60 \mathrm{~Hz}$ refresh rate) located outside the shielded room.

Data acquisition. MEG was recorded continuously during the runs (sampling rate: $1250 \mathrm{~Hz}$ ), using a 151-channel whole-head system (axial gradiometer, CTF System, Canada). We ensured that the head position relative to the sensors did not change by more than $5 \mathrm{~mm}$ along the whole experiment. Electro-oculogram was also measured for offline artefact rejection.

\section{B. MEG analyses}

Trials with signal artefacts (blinks, saccades, muscle signal...) were rejected using semiautomatic procedures. A 300-ms baseline was defined in the fixation period.

Event-related magnetic field $(E R F)$. Trials were averaged by task and type of target, resulting in six ERF by subject.

Source estimation. The cortical current density was estimated using overlapping spheres for the head model and a minimum-norm constraint for the solution of the inverse problem (http://neuroimage.usc.edu/brainstorm). Individual anatomy was available for half subjects; scalp tessellation was then computed using BrainVISA (http//brainvisa.info). For the other half, we used a default head model based on the same template as that used to define the cortical surface on which results were projected (MNI "Colin 27" brain).

Sensors and regions of interest (ROI). The response to the $3 \mathrm{D}$ target condition in the Presence task was used to delineate groups of sensors and brain areas activated by structure-from-motion perception. We selected four regions of interest (see Figure 2), the activity of which was representative of the profiles encountered in all activated regions.

ANOVA. ERF were averaged within sensor groups and analyzed time by time in a repeated-measure ANOVA with factors Task (Shape, Motion); Target (3D, Null) and Sensors (Occip, Temp, Pariet, Centr, Front). We also performed a 2 x 2 (Task, Target) ANOVA in the selected areas.
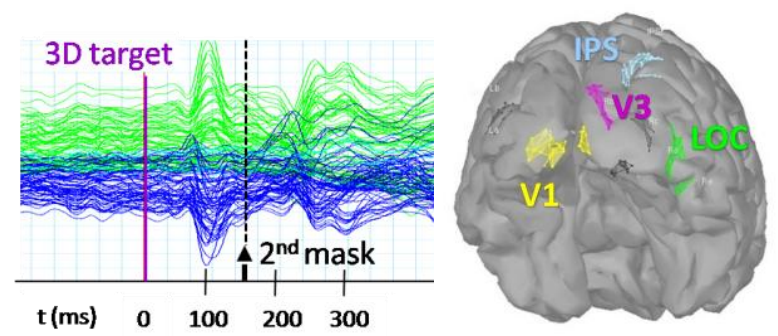

Fig. 2 Activity related to the 3D target in the Presence task (left) and selected regions of interest (right). V3: superior occipital gyrus; LOC: lateral occipital cortex; IPS: intraparietal sulcus.

\section{RESUltS}

\section{A. Temporal dissociation of task and target effects}

The ANOVA performed on the sensors revealed a temporal dissociation of the task and target effects, with significant ( $\mathrm{p}<0.001$ uncorrected) target $\mathrm{x}$ sensors and task $\mathrm{x}$ sensors crossed effects occurring respectively between 100 
and $200 \mathrm{~ms}$, and between 300 to $320 \mathrm{~ms}$. These crossed effects are explained by the topographies depending on the target in the first time window, and on the task in the second time window. More precisely, the target-related effect corresponds to modulations in the occipital sensors, whereas the task-related effect better corresponds to modulations in the parietal and temporal sensors.

\section{B. Cascade of activity from V1 to IPS}

At the source level, both the time window of the target effects and the activity profiles (see Figure 3 ) reveal the successive involvement of the regions of interest in processing the 3D stimulus.
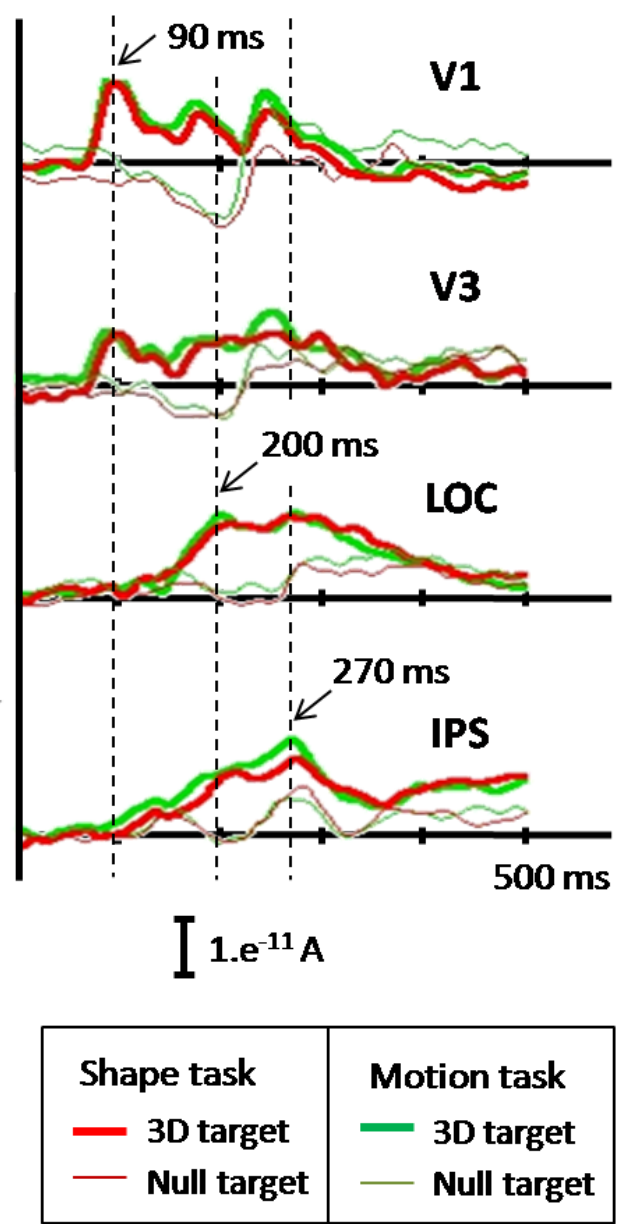

Fig. 3 Reconstructed activity in the regions of interest

The four areas indeed show very different time course after the 3D target, with tiered peak latencies. In V1, we observe a peak at $90 \mathrm{~ms}$ (M100 for target apparition) and another one at $240 \mathrm{~ms}$, well accounted for by the M100 of the $2^{\text {nd }}$ mask (onset at $150 \mathrm{~ms}$ ). In line with this, we find a target effect (with $\mathrm{p}<0.01$ uncorrected during more than 20 samples) during the first peak ( 85 to $190 \mathrm{~ms}$ ). We also find a late task effect during the M100 of the $2^{\text {nd }}$ mask (230 to $250 \mathrm{~ms}$ ). V3 also shows an early peak at $90 \mathrm{~ms}$ and corresponding target effects between 90 and $210 \mathrm{~ms}$. LOC, with a later peak at $200 \mathrm{~ms}$, shows target effects between 200 and 350 ms. Neither V3 nor LOC reach significant task effect. Eventually, IPS activity rises slowly after the beginning of the activity in LOC, peaks at $170 \mathrm{~ms}$, and falls back to the baseline prior the delayed motor response. It does not show any significant effect of target or task.

The activity related to the $2^{\text {nd }}$ mask, which is visible in the null target condition, reveals the same succession of activity in the different areas.

\section{DISCUSSION}

We recorded activity related to structure from motion integration in a mere detection task (Presence), as well as in identification tasks (Shape and Motion) requiring selective attention to the shape or 3D motion of the stimulus.

\section{A. Task-related modulation}

The regions of interest highlighted by the processing of SFM stimuli in the detection condition did not appear to be significantly modulated by the identification tasks, which is consistent with previous fMRI results [4] showing that the attentional selection of 3D motion and shape involve different areas from stimulus-driven activity. Rather, the task effect seems to involve more anterior visual brain regions as well as right fronto-polar areas. Further study is required to clarify whether task difficulty [5] or prospective memory due to the delayed response [6] might account for such frontal activity.

\section{B. From V1 to IPS}

In the regions of interest, the time window of the target effect, as well as the activity profiles, revealed the successive involvement of these areas in processing the 3D stimulus. This cascade being consistent with what has been described recently for structure from motion stimuli [7].

Note that we find the implication of the superior occipital gyrus (putative V3) at earlier latencies than LOC activation, consistent with our previous proposal that this area could be involved in an intermediate stage of gross shape extraction.

Although one can wonder to what extent spiking activity can be related to MEG signal, we find in IPS a late climbing activity similar to that described in cell studies [1]. Also, the particular timing of IPS activity relative to MT/LOC is consistent with the idea that IPS might accumulate evidence 
about the moving stimulus before reaching a perceptual decision, as long as information about the target is required, and available from earlier areas. Further studies modulating both the amount of information necessary to reach a decision and the possible duration of stimulus integration (between masks) would be needed to confirm the causal relation of the activity in these areas.

\section{Target-related activity and masking}

In this study, we used a time constrained paradigm with masks immediately preceding and following the target in order to segregate stimulus integration stages from task related-activity and hopefully perceptual decision. Our hypothesis however, was that the dynamical masks would successfully interfere with the integration of the visual cues leading to the perception of the 3D target stimulus. It is noteworthy, that contrary to [7], we neither found massive and sustained activity in the infero-temporal areas nor a rebound of activity in the early visual areas (V1 and V3) around $500 \mathrm{~ms}$. This is a first hint that target visual integration is interrupted by the masks.

Another indication is given by the response to the $2^{\text {nd }}$ mask after a Null target (Figure 4.A).

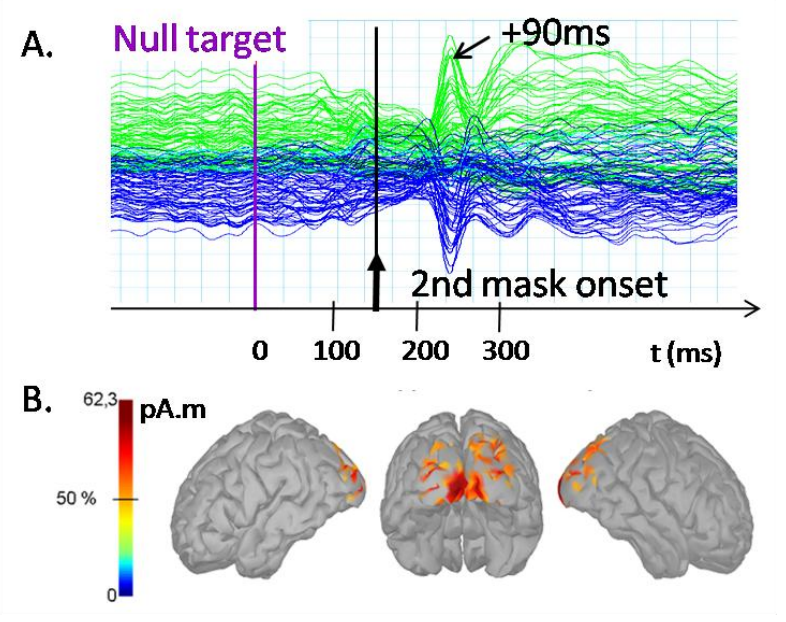

Fig. 4 Temporal window of target integration (A) and brain areas (B) defined by the response to the masks

In this condition, the mask-related M100 clearly appears in the ERF, delimiting the moment when target-related signal may not be observable anymore. Such response to the mask can also be found for the 3D target condition in V1, and to a less extent in V3 (see figure 3). Eventually, the sources of the response to the mask (Figure 4.B) appear to overlap largely with the areas involved in the $3 \mathrm{D}$ target processing, suggesting that the mask effectively compete with the $3 \mathrm{D}$ target for access to cascade of visual processing.

\section{v. Conclusions}

ERF revealed temporally non-overlapping effects of stimulus-driven and task-related processes in the perception of structure-from-motion stimuli.

Source reconstruction showed that the occipital, dorsal and ventral stimulus-related areas follow different temporal profiles and confirms a cascade of activity from V1 to IPS passing through MT/LOC for moving object perception.

The rising activity found in IPS may reflect accumulating evidence about the motion stimulus presented, in line with the results from single cell studies in monkey LIP [1].

\section{ACKNOWLEDGMENT}

This work was supported by a doctoral grant from DGA to AM and a MRIS-DGA grant (2006.34.059) to JL.

\section{REFERENCES}

1. Huk AC, Shadlen MN (2005) Neural activity in macaque parietal cortex reflects temporal integration of visual motion signals during perceptual decision making. J Neurosci 25 (45): 10420-36.

2. Kourtzi Z, Krekelberg B, van Wezel RJA (2008) Linking form and motion in the primate brain. Trends Cogn Sci 12: 230-236

3. Miskiewicz A, Buffat S, Paradis AL, Lorenceau J (2008) Shape and motion interactions at perceptual and attentional levels during processing of structure from motion stimuli. J Vis 8 (16): 17 1-14.

4. Paradis AL, Droulez J, Cornilleau-Peres V, Poline JB (2008) Processing 3D form and 3D motion: respective contributions of attentionbased and stimulus-driven activity. Neuroimage 43: 736-47.

5. Mangina CA, Beuzeron-Mangina H, Ricciardi E, Pietrini P, Chiarenza GA, Casarotto S (2009) Neural correlates of "analytical-specific visual perception" and degree of task difficulty as investigated by the Mangina-Test: a functional magnetic resonance imaging (fMRI) study in young healthy adults. Int J Psychophysiol 73: 150-6.

6. Burgess PW, Scott SK, Frith CD (2003) The role of the rostral frontal cortex (area 10) in prospective memory: a lateral versus medial dissociation. Neuropsychologia 41: 906-18.

7. Jiang Y, Boehler CN, Nonnig N, Duzel E, Hopf JM, Heinze HJ, Schoenfeld MA (2008) Binding 3-D object perception in the human visual cortex. J Cogn Neurosci 20: 553-62.

\author{
Corresponding author: \\ Anne-Lise Paradis \\ Cricm, équipe Cogimage - LENA \\ 47, bd de l'hôpital \\ F-75013, Paris, France \\ Email: anne-lise.paradis@upmc.fr
}

